\title{
GW23-e2282 A CLINICAL STUDY OF RETROGRADE GUIDEWIRE THERAPY IN CHRONIC CORONARY TOTAL OCCLUSION
}

doi:10.1136/heartjnl-2012-302920l.29

${ }^{1}$ Hong Lang, ${ }^{2}$ Wang Hong, ${ }^{3}$ Li Linfeng, ${ }^{3}$ Yin Qiulin, ${ }^{3}$ Hong Lang. ${ }^{1}$ The Second Department of Cardiology, Jiangxi Province People's Hospital; ${ }^{2}$ The Second Department of Cardiology, Jiangxi Province People's Hospital; ${ }^{3}$ The Second Department of Cardiology Jiangxi Province People's Hospital

Objectives To investigate the feasibility and safety of retrograde guidewire therapy in chronic coronary total occlusion (CTO).

Methods A total of 22 cases of coronary heart disease with CTO were treated from June 2008 to December 2011 in our hospital, aged 51-68 (59 \pm 7 ) years of age, 15 males and seven females. Coronary angiography showed coronary artery CTO, of which 14 were right coronary artery CTO, eight patients with left anterior descending coronary artery CTO. After a failed antegrade CTO recanalisation attempt, a retrograde approach via septal collaterals was tried. Success rate, procedure time, x-ray exposure time, contrast agent, complications and major adverse cardiac events were recorded (including cardiac death, myocardial infarction and target lesion revascularisation).

Results 20 patients underwent successful retrograde guidewire technique, with a the success rate of $90.9 \%$, procedure time 112 $226(162 \pm 35) \mathrm{min}, \mathrm{x}$-ray exposure time of $54-98(72 \pm 20) \mathrm{min}$, contrast agent $180-290(236 \pm 27) \mathrm{ml}$, without cardiac death and other serious complications. Patients were followed up for 336 months. Two patients had recurrent angina. Coronary angiography showed one case of restenosis, and the other non-target vessel stenosis.

Conclusions When the antegrade guidewire technique cannot be passed along CTO, the retrograde guidewire therapy is feasible and safe, can increase the technical success rate of CTO recanalisation attempts. The success rate of retrograde guidewire technique is best for patients with good heart and kidney function, due to the longer procedure time and larger volumes of contrast needed. The interventional cardiologist must also be prepared to spend more time on the procedure. 